\title{
A atuação do enfermeiro no tratamento de mulheres com neoplasia mamária: uma
}

\section{revisão de literatura}

\author{
The role of nurses in the treatment of women with breast cancer: a literature review
}

El papel de las enfermeras en el tratamiento de mujeres con cáncer de mama: una revisión de la

literatura

Recebido: 27/09/2021 | Revisado: 07/10/2021 | Aceito: 14/10/2021 | Publicado: 17/10/2021

\author{
Roseli Lucri \\ ORCID: https://orcid.org/0000-0001-6250-0164 \\ Faculdade Cristo Rei, Brasil \\ E-mail: roselilk72@gmail.com \\ Marli de Oliveira Costa \\ ORCID: https://orcid.org/0000-0002-9616-4477 \\ Faculdade Cristo Rei, Brasil \\ E-mail: marli@faccrei.edu.br
}

\begin{abstract}
Resumo
O presente estudo objetivou mostrar a assistência dada pelo enfermeiro às mulheres acometidas pela neoplasia mamária. Essas mulheres são acompanhadas e orientadas em seus tratamentos por uma equipe multiprofissional oncológica, porém, é o enfermeiro que se encontra diretamente ligado às pacientes prestando atendimento e acompanhamento na prática diária com os cuidados na cancerologia. Foi sobre a atuação deste profissional que se desenvolveu este estudo. A metodologia utilizada foi pesquisa bibliográfica com revisão na literatura, do tipo narrativa. A busca foi realizada a partir de consultas às bases de dados Scielo, Medline (PubMed) e Lilacs (BVS). O levantamento foi realizado com os seguintes descritores: "Neoplasia mamária" $A N D$ e $O R$ "câncer de mama" $A N D$ e $O R$ "Papel do enfermeiro" $A N D$ e, $O R$ "Oncologia". A busca totalizou 295 estudos, aplicando os critérios de tempo (2000/2020) e publicações em Português, refinou-se para 48, que desses, foram selecionados 34 . Os resultados encontrados mostraram que o enfermeiro traz um papel primordial no tratamento e acompanhamento às pacientes com diagnóstico de neoplasia mamária nas redes de atenção à saúde, seja na atenção primária, segundaria ou terciaria e também no pós-morte. Assim, a conclusão geral é que o enfermeiro é uma peça fundamental da equipe de enfermagem que integra a multiprofissional, pois a ele cabe a responsabilidade de instituir uma interação entre a paciente diagnosticada com a neoplasia mamária e seu tratamento através de uma comunicação terapêutica, e ao mesmo tempo propiciar uma assistência de enfermagem que atenda suas expectativas e necessidades, proporcionando bem-estar físico, emocional e espiritual.
\end{abstract}

Palavras-chave: Neoplasia mamária; Câncer de mama; Papel do enfermeiro; Oncologia.

\begin{abstract}
This study aimed to show the assistance given by nurses to women affected by breast cancer. These women are monitored and guided in their treatments by a multidisciplinary oncology team, however, it is the nurse who is directly linked to the patients, providing care and monitoring in daily practice with care in cancerology. It was about the performance of this professional that this study was developed. The methodology used was bibliographical research with literature review, of the narrative type. The search was performed based on queries to the Scielo, Medline (PubMed) and Lilacs (BVS) databases. The survey was carried out with the following descriptors: "Breast neoplasm" AND and OR "breast cancer" AND and OR "Nurse's role" AND and, OR "Oncology". The search totaled 295 studies, applying the criteria of time (2000/2020) and publications in Portuguese, it was refined to 48, of which 34 were selected. patients diagnosed with breast cancer in health care networks, whether in primary, secondary or tertiary care and also in the post-mortem. Thus, the general conclusion is that the nurse is a fundamental part of the nursing team that integrates the multiprofessional, as he/she is responsible for instituting an interaction between the patient diagnosed with breast cancer and her treatment through therapeutic communication, and at the same time, providing nursing care that meets their expectations and needs, providing physical, emotional and spiritual well-being.
\end{abstract}

Keywords: Breast neoplasm; Breast cancer; Nurse's role; Oncology.

\section{Resumen}

Este estudio tuvo como objetivo mostrar la asistencia brindada por enfermeras a mujeres afectadas por cáncer de mama. Estas mujeres son monitoreadas y guiadas en sus tratamientos por un equipo de oncología multidisciplinar, sin embargo, es la enfermera quien está directamente vinculada a las pacientes, brindando atención y seguimiento en la práctica diaria 
con atención en cancerología. Fue sobre el desempeño de este profesional que se desarrolló este estudio. La metodología utilizada fue la investigación bibliográfica con revisión de la literatura, de tipo narrativo. La búsqueda se realizó en base a consultas a las bases de datos Scielo, Medline (PubMed) y Lilacs (BVS). La encuesta se realizó con los siguientes descriptores: "Neoplasia de mama" Y y O "cáncer de mama" Y y O "Función de la enfermera" Y y, O "Oncología". La búsqueda totalizó 295 estudios, aplicando el criterio de tiempo (2000/2020) y publicaciones en portugués, se refinó a 48, de los cuales se seleccionaron 34. pacientes diagnosticadas de cáncer de mama en redes de atención de salud, ya sea en primaria, secundaria o terciaria cuidado y también en la autopsia. Así, la conclusión general es que el enfermero es parte fundamental del equipo de enfermería que integra al multiprofesional, pues es el encargado de instituir una interacción entre la paciente diagnosticada de cáncer de mama y su tratamiento a través de la comunicación terapéutica, y al mismo tiempo. tiempo, brindando cuidados de enfermería que satisfagan sus expectativas y necesidades, brindándoles bienestar físico, emocional y espiritual.

Palabras clave: Neoplasia de mama; Cáncer de mama; Papel de la enfermera; Oncología.

\section{Introdução}

Buscou-se nessa linha de pesquisa, ressaltar a assistência que o profissional da enfermagem realiza nos tratamentos da neoplasia mamária em mulheres. Desta forma, este estudo se mostrou pertinente na significação de registros científicos que possibilitem evidenciar a atuação do enfermeiro na prevenção, trato e cuidados frente aos danos que esse tipo de tratamento ocasiona.

De acordo com pesquisas realizadas pelas Agência Internacional para Pesquisa em Câncer (IARC) e seu projeto Global Câncer (GLOBOCAN, que avalia estimativas de incidência e mortalidade por câncer, através de dados de cada país/região) a nível mundial, a taxa de crescimento dos índices de câncer atingiu $20 \%$ na última década e o impacto do câncer corresponderá a $80 \%$ na população entre os países desenvolvidos e em desenvolvimento (Fretta et al., 2019).

É expressivo o número de pessoas que morrem por determinados tipos de câncer e embora essa estatística varie ao redor do globo, uma análise realizada em 2012 pela IARC, mostrou que nos diversos continentes, os cânceres com maiores índices de mortalidade masculina foram o de pulmão, próstata, estômago e colorretal. Já, no que se refere à mortalidade feminina, as taxas mais altas, vêm em primeiro lugar o de câncer de mama, depois, pulmão, colorretal, colo do útero e estômago. (Carvalho \& Paes (2019).

Em 2020, o IARC realizou outro estudo, que mostrou que o câncer de mama ainda é o mais incidente em mulheres no mundo, com aproximadamente 2,3 milhões de novos casos em 2020, o que representa 24,5\% dos casos novos por câncer em mulheres. É também a causa mais frequente de morte por câncer nessa população, com 684.996 óbitos estimados para esse ano (15,5\% dos óbitos por câncer em mulheres). (IARC, 2020). Portanto, o câncer de mamar ainda é considerado um problema de saúde pública de nível mundial. (Ambrósio \& Santos, 2015).

O que não se difere no Brasil, que de acordo com o Instituto Nacional de Câncer José Alencar Gomes da Silva (INCA), o câncer de mama, é um dos tipos de tumores que mais afetam as mulheres hoje em dia no país, estimam-se 66.280 novos casos de câncer de mama, para cada ano do triênio 2020-2022, o que corresponde a um risco estimado de 61,61 novos casos a cada 100 mil mulheres (INCA, 2020). O câncer de mama não tem uma causa única, pois diversos fatores podem estar relacionados ao aumento do risco de desenvolver a doença. Os fatores de risco envolvem fatores genéticos/hereditários, hormonais/endócrinos e ambientais/comportamentais. (Sartori \& Basso (2019).

Esses fatores de risco, em sua maioria, encontram-se relacionados à saúde da mulher e podem se estabelecer ao desenvolvimento do câncer de mama, como a idade, menarca precoce, nuliparidade, idade da primeira gestação a termo acima dos 30 anos, anticoncepcionais orais, menopausa tardia, terapia de reposição hormonal, ingestão de bebida alcoólica, sobrepeso e obesidade, inatividade física, exposição à radiação ionizante entre outros. No entanto, dentre esses fatores de risco, a idade continua sendo um dos mais relevantes, ou seja, mulheres mais velhas, particularmente a partir de 50 anos de idade, têm maior 
risco de desenvolver o câncer de mama, devido ao acúmulo de exposição ao longo da vida e as próprias alterações biológicas, que com o envelhecimento aumentam, esse risco (Silva \& Silva, 2005, INCA, 2011, 2020).

Neste contexto, o câncer de mama é uma das doenças que mais desencadeiam medo e angústia entre as mulheres em razão das altas taxas de incidência e mortalidade pontuado em pesquisas, e ter o suporte e a assistência da equipe de enfermagem, pode contribuir para que a mulher receba o diagnóstico do câncer de mama e enfrente o tratamento oncológico com menores danos e maior acolhida. Logo, o enfermeiro torna-se um importante suporte para a mulher que está vulnerável e doente, pois são esses profissionais que se encontram em constante contato com a paciente oncológica pelo fato da alta permanência no ambiente hospitalar e em virtude dos retornos estabelecidos pelos protocolos de tratamento, acarretando uma dependência dos cuidados profissionais e humanos do enfermeiro (Balsanelli \& Grossi, 2016).

De forma geral, o câncer ainda é entendido pelas pessoas como um processo de dor, sofrimento e morte. Diante dessa concepção, compete ao enfermeiro identificar suas próprias perspectivas diante da doença e determinar estratégias de enfrentamento e cuidados, sempre norteado pela legislação que ampare o exercício da função como as Resoluções do Conselho Federal de Enfermagem (COFEN). Embasados nessa vertente, o enfermeiro oncológico respalda-se nas Resoluções COFEN ${ }^{\circ}$ 210/1998 (Dispõe sobre as Normas Técnicas de Biossegurança Individual, Coletiva e Ambiental dos procedimentos a serem realizadas pelos profissionais de Enfermagem que trabalham com quimioterapia antineoplásica; no COFEN nº 211/1998 (Dispõe sobre as Normas Técnicas de radioproteção nos procedimentos a serem realizados pelos profissionais de Enfermagem que trabalham com radiação ionizante em Radioterapia, Medicina Nuclear e Serviços de Imagem) e COFEN nº 358/2009 (Dispõe sobre a Sistematização da Assistência de Enfermagem e a implementação do Processo de Enfermagem em ambientes, públicos ou privados, em que ocorre o cuidado profissional de Enfermagem) para proporcionar uma assistência adequada, eficaz e humanizada que permita minimizar o sofrimento das pacientes e familiares envolvidos (Mineo et al.,2013).

Diante desse contexto, enfocando fundamentalmente a atuação do enfermeiro em ambientes específicos de tratamento de mulheres com diagnóstico de câncer de mama, o objetivo principal deste trabalho de pesquisa foi mostrar a assistência dada pelo enfermeiro às mulheres acometidas pela neoplasia mamária. E os objetivos específicos mostraram uma breve explicativa sobre a neoplasia mamária, causas, consequências e prevenção na função do enfermeiro; a explanação dos cuidados e assistência do trabalho que é desenvolvido pela equipe de enfermagem, que se inclui as especialidades da enfermagem na prática dos cuidados na cancerologia; e especificar sobretudo, a atuação do enfermeiro, e os cuidados que podem ser realizados em qualquer ambiente de cuidados de saúde, desde residências e comunidades até instituições de cuidados agudos e centros de reabilitação, contemplando a assistência por redes de atenção à saúde em seus pontos de atuação (primaria, secundária e terciaria). Foram a partir desses pressupostos que norteou-se e desenvolveu-se este trabalho de pesquisa.

\section{Metodologia}

Tratou-se de uma pesquisa bibliográfica com revisão da literatura do tipo narrativa. A busca foi realizada em duas etapas: uma, foi por meio de fontes primárias como livros, teses e dissertações e, periódicos eletrônicos hospedados em bibliotecas virtuais de universidades brasileiras com os descritores: neoplasia mamária, câncer de mama, papel do enfermeiro e oncologia. E, a outra etapa foi a partir de dados secundários com consultas às bases de dados Scielo, Medline (PubMed) e Lilacs (BVS), onde o levantamento foi realizado com os mesmos descritores, só que utilizando-se de operadores Booleanos: "Neoplasia mamária" $A N D$ e $O R$ "câncer de mama" $A N D$ e $O R$ "Papel do enfermeiro" $A N D$ e, $O R$ "Oncologia".

Segundo Gil (2002) e Severino (2007) na realização da pesquisa bibliográfica o pesquisador tem que ler, refletir e escrever o sobre o que estudou, se dedicar ao estudo para reconstruir a teoria e aprimorar os fundamentos teóricos. Uma pesquisa bibliográfica tem como objetivo reunir textos publicados para serem analisados e servirem de apoio ao trabalho científico, ou 
seja, é o levantamento ou revisão de obras publicadas sobre a teoria que irá direcionar o trabalho científico, necessitando de dedicação, estudo e análise pelo pesquisador que irá executar o trabalho científico. (Gil, 2002).

Segundo Marconi \& Lakatos (2010), a revisão de literatura narrativa não utiliza metodologia definida para seu desenvolvimento, ficando a cargo dos autores a identificação e seleção de estudos, sua análise e interpretação. Seu processo de desenvolvimento constitui-se da identificação, localização, compilação, análise e interpretação do conhecimento de fontes diversas como livros, artigos, relatórios, dissertações e teses onde os diferentes argumentos adquiridos são apresentados pelo pesquisador em uma estrutura narrativa.

\subsection{Critérios de analise, inclusão e exclusão}

No cômputo geral, a busca totalizou 295 publicações, pois a temática é abrangente em diversas áreas da saúde, onde utilizou-se como critério de análise o referencial teórico, na abordagem aos cuidados da enfermagem/enfermeiro no tratamento ao câncer de mama em mulheres. Os critérios de inclusão abrangeram estudos publicados nos últimos 20 anos (2000/2020) e em português, e estudos que tivessem referência especifica sobre diagnóstico, tratamento, prevenção e atendimento da neoplasia mamaria em mulheres e cuidados de enfermagem.

Os critérios de exclusão, envolveram todas as publicações que não se enquadraram no critério temporal delimitado (2000/2020), e que não faziam referência direta à atuação do enfermeiro em tratamentos oncológicos com mulheres e que não tivessem informações relevantes aos objetivos da pesquisa. A partir daí, refinou-se para 48 estudos, desses, foram selecionados 34, sendo 23 artigos de bases de dados e 11 publicações científicas por meio de livros e periódicos eletrônicos hospedados em bibliotecas virtuais, para compor o referencial teórico que embasou este projeto de pesquisa.

Também utilizou-se neste estudo, registros documentais norteadores dos procedimentos da enfermagem para informações de marco legal, contidas no site do Conselho Federal de Enfermagem (COFEN) e sites com publicações de estatísticas sobre taxa de crescimento, índices, estimativas e perspectivas de câncer na população global como a Agência Internacional para Pesquisa em Câncer (IARC), projeto Global Câncer (GLOBOCAN), assim como, estatísticas realizadas no Brasil com informações referentes à população feminina acometida pelo câncer de mama, como o Instituto Nacional de Câncer José Alencar Gomes da Silva (INCA), Ministério da Saúde (MS), Sistema Único de Saúde (SUS), Unidade de Assistência de Alta Complexidade em Oncologia (UNACON) e Centro de Assistência de Alta Complexidade em Oncologia (CACON), que se encontram em estabelecimentos de saúde habilitados a oferecer assistência especializada e integral ao paciente com câncer.

\section{Resultados e Discussão}

Os estudos selecionados se encontram relacionados no referencial bibliográfico, separados por blocos: fontes primárias, secundárias e documental, onde cada bloco encontra-se em ordem crescente de autor/data. O resultado e as discussões, deram-se mediante os critérios de análise e os objetivos propostos nesse estudo.

\subsection{A Assistência da Enfermagem Oncológica}

Neoplasia ou câncer são palavras usadas para representar tumores malignos localizados em diferentes tecidos e regiões do organismo, assim, os gradativos níveis da mortalidade por câncer têm colocado essa doença como a segunda principal causa de morte no mundo, principalmente em países pobres e em desenvolvimento. Projeções apontam que se continuar assim nessa ascensão, nas próximas décadas virá ocupar o primeiro lugar nesse ranking (Bray et al., 2012, Mineo et al., 2013).

O INCA estimou que no ano de 2010 os diagnósticos de câncer de mama chegariam a cerca de 49.240 novos casos em todo o país, estimativa essa, que viria a se confirmar posteriormente (Brasil, 2010). Assim, o INCA recomenda a utilização de 
alguns tratamento contra o câncer de mama, embora cada caso seja específico e com indicações distintas, mas a regra geral, as mais utilizadas são as cirurgias, quimioterapia, radioterapia e hormonioterapia ${ }^{1}$.

Segundo Mineo et al. (2013) o estímulo ao uso de uma terapêutica apropriada aliado a um tratamento multidisciplinar, pode definir estratégias que priorizem a descoberta precoce e consequentemente, o seu controle. No entanto, mesmo com o esforço e o envolvimento do Ministério da Saúde em campanhas de prevenção precoce e no incentivo ao autoexame das mamas pelas mulheres, assim como a mamografia anualmente, o câncer de mama ainda é considerado a principal causa de morte por câncer, entre as mulheres no Brasil. Desta forma, o diagnóstico precoce do câncer de mama permite grandes probabilidades de cura, possibilitando tratamentos da própria mama e muito menos agressivos. (Spence, Johnson, 2012).

O tratamento para o câncer de mama deve ser realizado por uma equipe multidisciplinar (médicos, enfermeiro, técnicos e auxiliares de enfermagem, psicólogos etc.) buscando um atendimento integral da paciente. As modalidades de tratamento mais comuns, incluem cirurgia, radioterapia, quimioterapia e hormonioterapia (Vieira, 2012, Sartori, Basso, 2019). As decisões terapêuticas são baseadas em parte no estadiamento, mas o tamanho do tumor, o tipo e o grau histológicos, o status linfonodal, os níveis dos receptores de estrogênio e progesterona no tecido tumoral, o status menopausal e as condições clínicas gerais da paciente são também imprescindíveis na instituição do tratamento adequado. Em geral, são usados dois ou três métodos de tratamento (Salvajoli, Souhami \& Faria, 2004).

Uma vez diagnosticado precocemente o câncer de mama, a chance de gerar um prognóstico pode possibilitar um tratamento cada vez mais eficaz e dessa forma a abordagem à paciente deve ser realizada de forma multiprofissional, visto que o câncer de mama afeta a mulher e sua feminilidade, seja pelos efeitos de uma quimioterapia ou pelas cicatrizes de uma cirurgia radical.

Diante desse fato, os profissionais de saúde que mantêm maior contato com pacientes oncológicos, são os da enfermagem e a eles cabe o papel de atendimento e apoio, por isso a impotência de trabalharem de forma humanizada, com base no conhecimento científico para que se tenha uma assistência de enfermagem sistematizada durante a detecção e tratamento do câncer, mantendo qualidade de vida das pacientes, durante todo o tratamento (Freire, Massoli, 2006).

Para Camargo e Souza (2003), a assistência de enfermagem em oncologia avançou muito desde seu aparecimento como especialidade. Os profissionais de enfermagem que já atuavam na área oncológica desempenhando papéis importantes no cuidado à paciente, com medidas de conforto para os casos cirúrgicos e/ou em tratamento paliativo para pacientes terminais, nos dias de hoje, atuam muito além dos cuidados técnicos (Stumm, Leite, Maschio, 2008).

Os autores complementam que com o surgimento de novos tratamentos contra o câncer de mama, surgiu a necessidade de realizar-se um trabalho multidisciplinar, direcionado não só para o cuidado, mas também para a pesquisa e para o atendimento psicológicos dos pacientes e seus familiares. Assim, inserir uma equipe de enfermagem no cuidado à paciente oncológica, exige conhecimentos, habilidades e responsabilidades, com objetivos claros e direcionadas à paciente, sua família e demais pessoas significativas, abrangendo aspectos físicos, emocionais, sociais e espirituais.

O que é corroborado por Recco, Luiz e Pinto (2005) ao defenderem que a prática de enfermagem em cancerologia deve incluir todas as especialidades da enfermagem, podendo ser realizada em qualquer ambiente de cuidados de saúde, sendo que a assistência de enfermagem pode ser prestada por qualquer um dos seus integrantes ou por todos, dependendo do caso, já

\footnotetext{
${ }^{1}$ A terapia hormonal é uma forma de terapia sistêmica, o que significa que atinge células cancerígenas em qualquer parte do corpo e não apenas na mama. É indicada para mulheres com tumores receptores hormonais positivos. Fonte: http://www.oncoguia.org.br/conteudo/hormonioterapia-para-cancer-de-mama/.
} 
que uma equipe em geral é formada pelo enfermeiro, técnicos e auxiliares de enfermagem, cada qual com suas atribuições especificas e legais.

O profissional da equipe de enfermagem da atenção primária e secundária tem a responsabilidade de aplicar em sua área assistencial seus conhecimentos sobre fatores de risco para o câncer de mama, medidas de prevenção da doença, por meio de mamografia e autoexame das mamas. A ele cabe orientar sobre os sinais e sintomas de alerta para o câncer, que percebidos com rapidez levam a um diagnóstico precoce e um prognóstico favorável a cura. Sua atuação também se dá no pré-operatório com pacientes que passam pela mastectomia, seja ela conservadora ou não (Sá, Souza, 2010).

Já, o profissional da equipe de enfermagem que atende a atenção terciária, se dedica a atender às necessidades dos pacientes que passam por tratamento complementar como a quimio prevenção, radioterapia e hormonioterapia (Sá, Souza, 2010). A assistência dada pelo enfermeiro deve seguir as atribuições que estão presentes no Decreto $n^{\circ}$ 94.406/87, que pormenoriza as ações de enfermagem em todos os campos que necessitam da sua assistência. Logo, a assistência oncológica deve ser voltada para o cuidado ao paciente de forma ampla e integral, valorizando prestação do cuidado em seu aspecto físico, social e emocional.

Para Costa e Chaves (2012), independente de qual atenção seja, primária, secundária ou terciária, cabe ao enfermeiro e sua equipe, exercer seu papel no controle da doença, com responsabilidade na avaliação diagnóstica, na intervenção e monitorização do tratamento por meio da comunicação com o paciente. Considerar o conjunto de fatores que interagem nos processos da doença e seus sintomas no paciente oncológico é um passo importante na interpretação do cuidado dos enfermeiros à paciente com câncer de mama.

\subsection{A Assistência por Rede de Atenção à Saúde}

É papel do enfermeiro a responsabilidade pela elaboração, execução e avaliação dos planos assistenciais de saúde dos pacientes atendidos, assim cabe a ele delegar encargos e tarefas de atenção primária, secundária e terciaria. A atenção primária é responsável por modificar ou eliminar fatores de risco para o câncer de mama, muito embora, a detecção precoce ainda é a melhor maneira de combater este tipo de neoplasia, pois só assim a doença adquire melhores chances de cura. (Carvalho et al, 2009).

Estudos apontam que alguns fatores ambientais ou comportamentais podem contribuir para aumentar os riscos de desenvolver o câncer de mama, no entanto, estudos epidemiológicos não fornecem evidências conclusivas que justifiquem a recomendação de estratégias específicas de prevenção primária, mas sabe-se que alguns fatores de risco como tabagismo e obesidade, devem ser alvo de ações direcionadas que possam promover à saúde e prevenção, não só do câncer, mas de doenças crônicas de forma geral (Brasil, 2008).

Qualquer mulher corre o risco de vir a desenvolver um câncer de mama, no entanto, alguns fatores podem tornar algumas mulheres mais vulneráveis que as outras, e é nesse ponto que a enfermagem primária pode atuar, auxiliando a minimizar esses fatores de risco e ao mesmo tempo, incorporar outros fatores de proteção na rotina de vida dessas mulheres. Assim, esses fatores de risco podem ser divididos em incontroláveis e controláveis (Mineo et al., 2013).

Os fatores incontroláveis podem ser: o sexo, já que mulheres são mais vulneráveis ao câncer de mama que os homens, por causa da exposição ao estrogênio; a idade da menarca e da menopausa; a exposição ao estrogênio; a raça, pois as mulheres brancas tendem a correr mais riscos que as de outras etnias, a nuliparidade e a genética, que é um dos fatores principais devido ao histórico familiar de câncer de mama.

Os controláveis pode ser: exposição a produtos químicos tóxicos e campos eletromagnéticos, tabagismo, obesidade, sedentarismo, alcoolismo, má alimentação (Leite, Oliveira, Ribeiro, 2007). Conhecedores dos fatores de risco controláveis e incontroláveis, a enfermagem trabalha na atenção primária visando reduzi-los por meio de orientações à população sobre a 
necessidade de uma vida mais saudável, com alimentação balanceada, exercícios físicos, evitando o tabagismo e alcoolismo e diminuindo a exposição a produtos tóxicos e campos eletromagnéticos.

Nesse mesmo viés, o profissional da enfermagem busca orientar as mulheres quanto ao diagnóstico precoce, principalmente para aquelas com histórico familiar de câncer de mama, incentivando o autoexame das mamas mensalmente, mamografia anualmente e consultas médicas periódicas nos serviços de saúde (Leite, Oliveira, Ribeiro, 2007, Brasil, 2008, Schwanke, Schneider, 2008).

Também é da sua competência segundo Barros (2008), explicitar os sinais e sintomas que podem ajudar a diagnosticar o câncer de forma precoce, orientando quanto ao uso abusivo de fármacos que contenham altas doses de estrogênios e também estimular o planejamento familiar para evitar gravidez tardia (Brasil, 2008). A assistência primária no câncer de mama é fundamental no estimulo ao exame clínico anual com a mamografia de rastreamento, acompanhada do autoexame das mamas mensalmente após o período menstrual. $\mathrm{O}$ autoexame estimula na mulher o hábito de conhecer o próprio corpo, e é na palpação da mama que a mulher consegue examinar o tecido mamário e linfonodos, estando atenta às alterações em suas mamas, já que em geral, os tumores mamários iniciais são pequenos e não provocam dor.

A Unidade Básica de Saúde (UBS) é o nível primário responsável pela orientação e detecção precoce do câncer de mama, sendo responsabilidade da equipe de enfermagem da UBS realizar reuniões educativas sobre o assunto, buscando a conscientização da comunidade sobre a importância da prevenção e detecção precoce do câncer de mama.

A responsabilidade da enfermagem na atenção secundária tem como foco a detecção precoce do câncer de mama através da mamografia diagnóstica, quando além do tratamento primário do câncer de mama, necessita de complementação, por suspeitas, sinais e sintomas de câncer. Desse modo, as crescentes e modernas tecnologias de diagnóstico, ampliaram a perspectiva de novos procedimentos e programas de prevenção secundária do câncer, pois quanto mais cedo for diagnosticado, maior será a chance de cura, de sobrevida e de qualidade de vida, além dos atendimentos e custos ser bem menores que os dos tratamentos mais longos (Brasil, 2008)

Segundo Barros (2008), é fundamental que haja uma assistência de enfermagem perioperatória, já que o tratamento primário do câncer de mama é a cirurgia, que pode ser conservadora ou não conservadora, dependendo do estadiamento e infiltração tumoral. Portanto, a mastectomia é um procedimento cirúrgico muito agressivo, que pode levar a consequências traumatizantes na vida e saúde da mulher. Por isso, a importância da comunicação dos profissionais de saúde, especialmente dos enfermeiros com a paciente durante todo o seu tratamento. A interação entre profissional e paciente, gera na paciente maior compreensão do que está acontecendo com ela, assim como, auxilia em como lidar com toda a situação estressante do tratamento.

A assistência e a atuação do enfermeiro na fase pré-operatória, vai ajudar a amenizar o desconforto e a implementar medidas que podem reduzir o medo e ansiedade pré e pós-operatória, pois cabe a esse profissional da enfermagem, promover a capacidade da paciente em tomar decisões e a incentivar o autocuidado, com o propósito de prevenir outras complicações nessa fase do tratamento (Mota, 2011).

Em suma, Alves et al. (2010) observam que quando há uma assistência de enfermagem humanizada e completa durante todo o pré-operatório, o pós-operatório costuma ser menos estressante, pois induz a paciente a descrever qualquer desconforto, sejam eles fisiológicos ou psicológicos, de menor ou maior intensidade. Da mesma forma, dá-se a assistência de enfermagem durante o período transoperatório, que é uma fase realizada com ações de enfermagem previamente planejadas e implementadas que vão desde a recepção da paciente na unidade de centro cirúrgico até a sua saída para a sala de recuperação anestésica, onde são verificados todos os efeitos pós-anestésicos, e monitoramento dos sinais vitais (Maia, 2008).

Ao enfermeiro, durante o período do transoperatório, cabe a responsabilidade de efetivar a prescrição de enfermagem feita no pré-operatório, monitorar todas as funções vitais do paciente, ficar atento aos monitores cardíacos, ventilação mecânica, dar suporte à equipe médica, verificar necessidade de instrumentação cirúrgica complementar, avaliação anestésica, observar 
manipulação dos instrumentos, limpeza e esterilização da sala cirúrgica e dos materiais, para diminuir e evitar infecção hospitalar (Maia, 2008).

Barreto et al. (2008) chamam a atenção para o pós-mastectomia, onde a equipe de enfermagem integrada à multidisciplinar deve estar atenta para complicações pós-cirúrgicas que poderão surgir, como: hemorragias, seroma, infecção, necrose da pele, diminuição do movimento dos braços devido à alguma lesão de nervo ou até mesmo, linfodema. Destarte, os procedimentos da enfermagem secundária ao aplicar os cuidados na recuperação da mulher em pós-operatório têm como principal objetivo prevenir complicações resultantes da cirurgia, dreno, reabilitação física e prioritamente, uma especial atenção para as questões relacionadas aos sentimentos e medo dessa paciente.

Corroborando, Carvalho et al. (2009) chamam esses cuidados de "valorização do autocuidado", onde consideram de fundamental importância a participação da própria mulher mastectomizada no processo de enfrentamento, prevenção de complicações, recuperação e reabilitação pós cirurgia. Porém não deixam de destacar como sendo essencial, o trabalho que a equipe de enfermagem desempenha nesses cuidados, trazendo um papel extremamente relevante em todo o processo de assistência e recuperação da paciente mastectomizada, pois são os promotores do suporte emocional e informativo dos cuidados necessários no pós-cirúrgico.

Em síntese, os cuidados e a assistência da enfermagem na atenção secundária traz um importante papel de apoio e suporte para as mulheres com diagnóstico de câncer de mama, já que pode haver um comprometimento da autoimagem nessas pacientes, gerando traumas de ordem física, emocional e social, o que consequentemente, também pode vir a influenciar de maneira negativa na recuperação e evolução do tratamento. Assim, a assistência da enfermagem deve promover conforto e tranquilidade frente a esses sentimentos e expectativas pelas quais a paciente passa, e igualmente, também orientar quando na alta, incentivar e direcionar a paciente para o autocuidado, assim como, a buscar grupos que promovam sua reintegração na sociedade e cotidiano familiar (Santos et al., 2011).

A mulher quando diagnosticada com câncer de mama, passa por uma série de enfrentamentos psicológicos e emocionais, principalmente quando vê alterada sua autoimagem pelos efeitos do tratamento ou algumas vezes destruída pela mastectomia. E tudo tende a se fragilizar quando há a necessidade de um tratamento complementar como uma quimioprevenção, radioterapia ou hormonioterapia, e nesse momento, a atuação da equipe de enfermagem na atenção terciária é primordial, pois torna-se um ponto de apoio substancial diante de uma paciente que na maioria das vezes se encontra abalada e desacreditada da cura, ou de uma chance de sobrevida (Stumm, Leite, Maschio, 2008).

A neoplasia mamária, em geral, abrange três etapas das quais a mulher vivencia forte impacto emocional: o recebimento do diagnóstico, a realização de um tratamento longo e agressivo e a aceitação de alterações em seu corpo que vão influenciam sua autoimagem para si e para os outros. Além dessas etapas, a mulher também tende a desenvolver sentimentos de medo, tristeza e negação, associados à importância da mama como símbolo de feminilidade, e pelo medo das reações dos tratamentos, principalmente, aos da quimioterapia.

Como os tratamentos tendem a deixar a mulher mais sensível e vulnerável aos efeitos colaterais, na maioria das vezes, isso acaba interferindo numa resposta eficaz ao tratamento, então é nesse momento que a atuação da equipe de enfermagem atua de forma efetiva, orientando quanto a esses efeitos quimioterápicos, dando suporte emocional à paciente e aos seus familiares (Vieira, Lopes, Shimo, 2007).

Enquanto não se consegue reverter o processo biológico que altera o comportamento da célula maligna, a quimioterapia continuará sendo usada como método auxiliar na tentativa de cura ou aumento de sobrevida em pacientes portadoras de carcinoma mamário. A quimioprevenção vem ganhando destaque, pois representa um avanço na cura e controle do câncer, aumentando a expectativa de vida do paciente (Melo et al., 2002). 
Desta forma, o enfermeiro oncológico se respalda na Resolução do COFEN 210/1998, que dispõe sobre a atuação dos profissionais de enfermagem que trabalham com quimioterápicos antineoplásicos, que retrata que é de competência do enfermeiro planejar, organizar, supervisionar, executar e avaliar todas as atividades de enfermagem, elaborar protocolos terapêuticos de prevenção, tratamento e minimização dos efeitos colaterais, realizar a consulta de enfermagem, ministrar o quimioterápico prescrito conforme a farmacocinética da droga e protocolo terapêutico e manter a biossegurança individual, coletiva e ambiental.(Brasil, 1998)

Frente a relevância do tratamento quimioterápico, existe a necessidade que a equipe de enfermagem possua conhecimentos específicos sobre os fármacos administrados e seus efeitos colaterais, assim como a conduta no extravasamento e tempo de infusão dos medicamentos empregados. Desta forma, enfermeiros que prestam assistência à pacientes oncológicos, trabalham num ambiente de grande exigência, seja ela pela alta complexidade da doença ou pelas manifestações de cada paciente, o que requer que esse profissional esteja em contínua inovação profissional (Stumm, Leite, Maschio, 2008).

Segundo o Brasil/INCA (2002), determinados tratamentos oncológicos necessitam da complementação da radioterapia, assim como no câncer de mama, onde funciona como quimioprevenção adjuvante, com a finalidade de combater células tumorais que ainda possam restar após a cirurgia, no entanto, nesse estágio do tratamento, a maioria das pacientes já se encontram exauridas das outras terapêuticas, como a cirurgia e a quimioterapia, já que a cada tratamento submetido gera novos diagnósticos, trazendo sentimentos de desamparo, angústia, medo, insegurança, independente da fase em que ela se encontra na terapia.

Assim, à vista disso, as consultas de enfermagem realizadas antes e durante o tratamento radioterápico, auxiliam sobremaneira na minimização destes problemas, com orientações e assistência de enfermagem integral (Araújo, Rosas, 2008). O enfermeiro na consulta radioterápica tem como responsabilidade identificar todos os problemas de enfermagem e definir quais são as necessidades básicas que foram afetadas e o grau de dependência da paciente. Prolongar a sobrevida de uma paciente é o objetivo de todo tratamento oncológico, então é substancial que o enfermeiro saiba identificar os problemas causados pelo tratamento e assim intervir juntamente com uma equipe multidisciplinar, auxiliando da melhor maneira possível, a paciente e seus familiares a passarem por mais uma fase do tratamento (Leal, Cubero, Giglio, 2010).

O papel do enfermeiro no cuidado pós-morte, está presente no Código de Deontologia dos Profissionais de Enfermagem, no art. $3^{\circ}$, no item que trata dos Princípios Fundamentais, onde diz: "o profissional de enfermagem respeita a vida, a dignidade e os direitos da pessoa humana em todo o seu ciclo vital, sem discriminação de qualquer natureza" (Brasil, 2003). Desta forma, a diferença elementar entre as pessoas em geral e os profissionais da área de saúde é que a vida e a morte já fazem parte do cotidiano destes profissionais, principalmente dos enfermeiros, que são aqueles que lidam diretamente com o paciente (Fernandes, Iglesiais, Avellar, 2009).

Assim sendo, os autores descrevem que havendo a constatação da morte de um paciente, a enfermagem deve realizar alguns procedimentos de cuidados com o corpo, sendo que a necessidade desses procedimentos vai estar associado ao que ocorreu com o paciente, como por exemplo fazer tamponamento dos orifícios, retirar todos os materiais e instrumentos que estavam nele, como cateteres, sondas e tubos respiratórios, e só depois, ele será encaminhado para a funerária.

Cabe destacar que o cuidado da enfermagem com o corpo após a morte deve resumir fundamentalmente na preservação do aspecto natural do ser humano, mas principalmente dar suporte emocional aos familiares, que mesmo com todo sofrimento no decorrer do tratamento contra o câncer, perder a luta e ainda ter que lidar com a perda deste ente querido (Fernandes, Iglesiais, Avellar, 2009). 


\subsection{Estatísticas e Informações sobre o Câncer de Mama}

Conforme dados do projeto Global Câncer (GLOBOCAN) realizado pela Organização Mundial da Saúde (OMS), o câncer foi responsável por 8,2 milhões de mortes (22\% de todas as mortes por doenças não transmissíveis) em 2012. Dessas, cerca de 4,7 milhões (57,3\%) e 3,5 milhões (42,7\%) foram de homens e mulheres, respectivamente, já no Brasil, as neoplasias ocupam o segundo lugar e foram responsáveis por 209.780 (16,6\%) óbitos em 2015. (Ferlay et al., 2012, Carvalho \& Paes, 2019). Dados do Instituto Nacional de Câncer (INCA) mostram que no ano de 2008 o número de mortes por câncer chegou a 11.860 óbitos, onde 11.735 eram mulheres e 125 eram homens. No entanto, dentre os cânceres mais temidos está o câncer de mama, uma doença heterogênea e complexa, que se apresenta de múltiplas formas clínicas e morfológicas, com diferenças na pré e pósmenopausa, diferentes graus de agressividade tumoral e potencial metastático. (Mineo et al, 2013).

Assim, nas palavras de Souza, Grando \& Couto Filho (2008) os atrasos no diagnóstico e início do tratamento aumentam consideravelmente o grau de ansiedade nas mulheres, o que pode impossibilitar ou dificultar tratamentos curativos e consequentemente, diminuir taxas de sobrevivência. Muito embora, os autores chamam a atenção para a história natural do câncer de mama preconizando que o desenvolvimento clínico da doença e a sobrevida pode variar muito de paciente para paciente, e estas variantes são estabelecidas por uma complexidade de fatores, tais como a velocidade de duplicação tumoral, o potencial de metastatização do tumor e outros mecanismos que podem estar relacionados com a condição imunológica, hormonal e nutricional do paciente. (Sartori \& Basso, 2019). Também, deve-se considerar a forma de vivencia da pessoa, já que estudos observacionais pontuam que o comportamento humano aliado ao estilo de vida, sedentarismo, falta de exercícios físicos e sem uma dieta balanceada gerando obesidade e/ou alcoolismo, podem favorecer o aumento da incidência do câncer de mama em todo o mundo (Vieira, 2012)

No Brasil em 2008, foi aprovada pelo Congresso Nacional a Lei n. 11.664 de 29 de abril (Brasil, 2008) e que entrou em vigor em 2010, garantindo às mulheres brasileiras a assistência pelo Sistema Único de Saúde (SUS) com a realização de exames confiáveis para a detecção do câncer de mama, como a mamografia anual a partir dos 40 anos, e, prioritamente a partir dos 50 anos, a mamografia com rastreamento, conforme mostra a Figura 1.

Segundo Brasil/INCA (2015), por ser uma doença heterogênea, o câncer de mama apresenta uma ampla variação de comportamento biológico, diferentes taxas de crescimento e potencial metastático distinto, entretanto os tumores de crescimento lento são mais facilmente detectados no rastreamento. Assim, o diagnóstico de câncer de mama em geral, baseia-se no exame de mamografia, já que possui comprovação científica da sua eficácia em detectar lesões pequenas que não conseguem ser detectadas por apalpação ou se estiverem em estádios iniciais. Sua detecção precoce deve ser enfatizada, cumprindo-se a lei brasileira ${ }^{\circ}$ 11.664, que garante o direito de todas as mulheres acima de 40 anos de realizar mamografia anualmente como rastreio para o câncer de mama (Sartori \& Basso, 2019).

De acordo com Mineo et al (2013), é importante saber que pacientes com diagnóstico confirmado de câncer de mama têm seu tratamento planejado de acordo com os exames complementares relacionados ao tumor e que são relevantes, como o estadiamento, receptores de estrógeno (RE), receptores de progesterona (RP), grau histológico e invasão vascular ou angiolinfática. 
Figura 1 - Rastreamento Mamográfico.

\begin{tabular}{|c|c|c|}
\hline \multicolumn{3}{|r|}{ MAMOGRAFIA } \\
\hline \multicolumn{2}{|c|}{ Questão norteadora } & $\begin{array}{l}\text { Qual a eficácia do rastreamento com mamografia na redução da mortalidade global e por câncer } \\
\text { de mama, comparada à ausência de rastreamento? }\end{array}$ \\
\hline \multirow{6}{*}{ 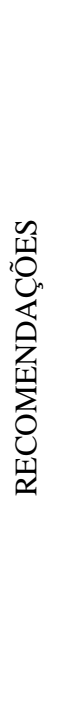 } & $<$ de 50 anos & $\begin{array}{l}\text { O Ministério da Saúde recomenda contra o rastreamento com mamografia em mulheres de } 50 \text { anos } \\
\text { (recomendação contrária forte; os possíveis danos superam os possíveis benefícios) }\end{array}$ \\
\hline & De 50 a 59 anos & $\begin{array}{l}\text { O Ministério da Saúde recomenda o rastreamento com mamografia em mulheres com idade entre } \\
50 \text { a } 50 \text { anos ( recomendação favorável fraca: os possíveis benefícios e danos provavelmente são } \\
\text { semelhantes) }\end{array}$ \\
\hline & De 60 a 69 anos & $\begin{array}{l}\text { O Ministério da Saúde recomenda o rastreamento com mamografia em mulheres com idade entre } \\
60 \text { a } 69 \text { anos (recomendação favorável fraca: os possíveis benefícios provavelmente superam os } \\
\text { possíveis danos) }\end{array}$ \\
\hline & De 70 a 74 anos & $\begin{array}{l}\text { O Ministério da Saúde recomenda contra o rastreamento com mamografia em mulheres com idade entre } \\
70 \text { e } 74 \text { anos (recomendação contrária fraca: o balanço entre possíveis danos e benefícios é incerto) }\end{array}$ \\
\hline & 75 anos ou mais & $\begin{array}{l}\text { O Ministério da Saúde recomenda contra o rastreamento com mamografia em mulheres com } 75 \text { anos } \\
\text { ou mais (recomendação contraria forte: os possíveis danos provavelmente superam os possíveis } \\
\text { benefícios) }\end{array}$ \\
\hline & Periodicidade & $\begin{array}{l}\text { O Ministério da Saúde recomenda que a periodicidade do rastreamento com mamografia nas faixas } \\
\text { etárias recomendadas seja bienal (recomendação favorável forte: os possíveis benefícios } \\
\text { provavelmente superam os possíveis danos quando comparada às periodicidades menores do que a } \\
\text { bienal) }\end{array}$ \\
\hline
\end{tabular}

Fonte: Brasil/INCA (2015, p.13).

Complementando, Vieira (2012) retrata que pode-se acompanhar o estadiamento do câncer de mama, que se inicia no exame físico, incluindo avaliação da pele, glândulas mamárias e dos linfonodos axilares, supraclaviculares e cervicais; sequencialmente, segue-se com métodos de imagem e dosagens sanguíneas de desidrogenase lática (DHL) e fosfatase alcalina (FA), até exames histopatológicos da mama ou de outros tecidos, para diagnóstico de tumor primário (T), linfonodos(N) e Metástase(M),conforme descrito na figura 2. Assim, o tratamento de câncer de mama pode envolver cirurgia (mastectomia) e a quimioprevenção, e em alguns casos a radioterapia e hormonioterapia. A conduta médica de escolher a cirurgia como primeiro passo no tratamento é essencialmente seletiva, pois cada paciente diagnosticada apresenta graduação diferente de desenvolvimento tumoral. 
Figura 2 - Estadiamento do câncer de mama.

$\begin{array}{ll}\text { Tumor Primário }(\mathbf{T}) \\ \text { Tis } & \text { Carcinoma intraductal, carcinoma lobular in situ } \\ \text { T1 } & \text { Tumor menor ou igual a } 2 \mathrm{~cm} \text { em sua maior dimensão } \\ \text { T1a } & \text { Tumor até } 0,5 \mathrm{~cm} \\ \text { T1b } & \text { Tumor de } 0,6 \text { a } 1 \mathrm{~cm} \\ \text { T1c } & \text { Tumor de } 1,1 \text { a } 2 \mathrm{~cm} \\ \text { T2 } & \text { Tumor de } 2,1 \text { a } 5 \mathrm{~cm} \\ \text { T3 } & \text { Tumor maior de } 5 \mathrm{~cm} \\ \text { T4 } & \text { Tumor de qualquer diâmetro com invasão direta da pele ou caixa torácica } \\ \text { T4a } & \text { Extensão à parede torácica } \\ \text { T4b } & \text { Edema (incluindo peau d'orange) ou ulceração da pele da mama, ou nódulos cutâneos } \\ & \text { satélites confinados à mesma mama } \\ \text { T4c } & \text { Associação de T4a e T4b } \\ \text { T4d } & \text { Carcinoma inflamatório } \\ \text { Linfonodos (N) } \\ \text { NX } & \text { Os linfonodos não podem ser avaliados (remoção cirúrgica previa) } \\ \text { N0 } & \text { Ausência de metástase para linfonodos } \\ \text { N1 } & \text { Acometimento de linfonodos axilares ipsilaterais móveis } \\ \text { N2 } & \text { Acometimento de linfonodos axilares ipsilaterais fixos } \\ \text { N3 } & \text { Acometimento de linfonodos da cadeia mamária interna ipsilateral } \\ \text { Metástases (M) } & \\ \text { M0 } & \text { Sem metástases à distancia } \\ \text { M1 } & \text { Metástases à distância } \\ & \end{array}$

Logo, a escolha do tipo de cirurgia vai depender da classificação do tumor e seu estadiamento clínico, já que na maioria das vezes a intervenção cirúrgica objetiva o controle da difusão do câncer para além dos tecidos mamários (Salvajoli, Souhami \& Faria, 2004, Vieira, 2012, Mineo et al., 2013). De acordo com Vieira (2012), a cirurgia conservadora (tumorectomia ou setorectomia), associada à pesquisa de linfonodo sentinela, é o tratamento padrão para todas as pacientes em estádios precoces, já a quimioterapia neoadjuvante é reputada para pacientes com tumores maiores, pois seu objetivo é reduzir o volume tumoral, proporcionando a preservação da mama pós-cirúrgica.

Segundo Salvajoli, Souhami \& Faria (2004), a radioterapia curativa é utilizada quase sempre em conjunto com a cirurgia, seja como tratamento adjuvante após a mastectomia, ou no tratamento conservador após a tumorectomia. As áreas que podem ser irradiadas no câncer de mama, com intenção curativa, são:

- a mama residual, após cirurgia conservadora, ou o plastrão mamário, após mastectomia;

- $\quad$ as drenagens linfáticas das fossas supra e infraclaviculares;

- $\quad$ linfonodos da axila, e

- cadeia da mamária interna

O tratamento pode ser feito de várias formas e como em boa parte da radioterapia, há sempre um componente subjetivo e pessoal nos limites dos campos de irradiação, muito embora cerca de metade dos pacientes submetidos a qualquer tratamento oncológico, inclusive o de câncer de mama, acabam fazendo uso da radioterapia em algum momento evolutivo da doença. Outro tipo de tratamento utilizado para o controle e a melhora na qualidade de vida de mulheres com câncer de mama, é a hormonioterapia paliativa, que é um recurso altamente eficaz na terapêutica complementar, pois tem bom perfil de toxicidade para as células malignas e proeminente eficácia. O tratamento intenta diminuir as taxas de estradiol que é um tipo de estrogênio, maléfico as células e também responsável pela formação de tumores malignos (Mineo et al., 2013).

É importante compreender que a idade e os fatores de risco relacionados à vida reprodutiva da mulher estão fortemente ligados ao desenvolvimento do câncer de mama, e desta forma, a Política Nacional de Atenção Oncológica garante o atendimento 
integral a qualquer doente com câncer, por meio das Unidades de Assistência de Alta Complexidade em Oncologia (UNACON) e dos Centros de Assistência de Alta Complexidade em Oncologia (CACON). Segundo a Portaria n ${ }^{\circ} 874$ de 16 de maio de 2013, este é o nível da atenção capacitado para determinar a extensão da neoplasia (estadiamento), tratar, cuidar e assegurar a qualidade dos serviços de assistência oncológica (Mineo et al., 2013).

\section{Considerações Finais}

Este estudo demonstrou que apesar de apresentar altas taxas de incidência, o câncer de mama pode ser curável numa parcela expressiva de pacientes, e que isso se deve essencialmente ao diagnóstico precoce pela mamografia e a valência do tratamento complementar com quimioterapia, radioterapia e/ou hormonioterapia. Os resultados encontrados na literatura mostram que mulheres acometidas pela neoplasia mamária enfrentam uma sucessão de consequências físicas e emocionais, pois o tratamento desperta sentimentos negativos, preconceitos e até a possibilidade de se deparar com a morte.

No entanto, quando a equipe de enfermagem que acompanha a paciente nessa luta for ativa e atuante, a parceria fará uma grande diferença na vida dessa mulher, e proporcionará mais força e crença na possibilidade da cura, trazendo maiores esperanças para a paciente e sua família. A enfermagem oncológica está presente em todas as fases pelas quais a paciente com neoplasia mamária passa ao longo de todo o processo terapêutico, desde o diagnóstico positivo até a reabilitação dos possíveis casos, e até mesmo no óbito e o pós-morte. Assim, o cuidado oncológico se aplica desde a atenção primária, secundária, terciária até o pós-morte como em qualquer outra área de atuação.

A assistência da enfermagem representada pela equipe formada pelo enfermeiro, técnicos e auxiliares de enfermagem, com suas atribuições pautadas e definidas pela legislação, onde o enfermeiro é o profissional responsável pela elaboração, execução e avaliação dos planejamentos assistenciais de saúde, trazem um papel fundamental no tratamento de cuidados à pacientes com câncer, já que atuam frente às equipes médicas com o objeto de trabalharem juntos na prevenção e controle de possíveis danos à saúde da paciente.

Diante dos resultados encontrados na literatura, percebeu-se quão fundamental é a atuação do enfermeiro no cenário oncológico como suporte no tratamento da neoplasia mamária. Também, faz-se necessário que haja juntamente, para maiores êxitos no combate à doença, a atuação da equipe de enfermagem em conjunto com a equipe multiprofissional para alcançar um desempenho multidisciplinar, com um acompanhamento especializado na área médica, uma comunicação entre os diversos profissionais da saúde e principalmente a presença constante do enfermeiro, com uma assistência sistematizada e humanizada.

O que foi perceptível nesse estudo, é que o cuidado da enfermagem oncológica se encontra na maior parte do tratamento direcionada à um apoio psicológico, assistindo a paciente de forma integral, conforme dispõe os vários documentos norteadores da conduta do enfermeiro o COFEN, e as disposições legais da profissão. É notório que o enfermeiro é uma peça fundamental da equipe multiprofissional, pois a ele cabe a responsabilidade de instituir uma interação entre a paciente diagnosticada com um câncer de mama e seu tratamento através de uma comunicação terapêutica, e ao mesmo tempo proporcionar uma assistência de enfermagem que atenda suas expectativas e necessidades, oportunizando conforto físico, emocional e espiritual.

Espera-se com esse estudo possa contribuir para evidenciar a atuação e o desempenho do profissional da enfermagem, no papel do enfermeiro, quando frente à assistência dada a mulheres em tratamento da neoplasia mamária, suscitando que possa haver novas pesquisas sobre a temática que venham a agregar e contribuir para a atuação desse profissional e que possibilitem evidenciar sua atuação na prevenção, trato e cuidados aos danos que esse tipo de tratamento ocasiona. 
Research, Society and Development, v. 10, n. 13, e382101321147, 2021

(CC BY 4.0) | ISSN 2525-3409 | DOI: http://dx.doi.org/10.33448/rsd-v10i13.21147

\section{Referências}

\section{Fontes Primárias}

Barros, A.C.D. (2008). Diagnóstico e Tratamento do Câncer de Mama. Sociedade Brasileira de Mastologia,. Brasília.

Bray, F. et al. (2012). Transições do câncer global de acordo com o Índice de Desenvolvimento Humano (2008-2030): um estudo de base populacional. Lancet, 13(8), 790-801.

Ferlay J. et al.( 2013). GLOBOCAN 2012: Estimativa de incidência, mortalidade e prevalência de câncer em todo o mundo, (10) [serial on the Internet]. IARC Cancer Base ,11. Recuperado de http://www.globocan.iarc.fr.

Freire, C. A.; \& Massoli, S. E. (2006). A assistência de enfermagem às pacientes com câncer de mama em tratamento quimioterápico. Batatais-SP: Centro Universitário Clarentino.

Leite, R. C.; Oliveira, C.; \& Ribeiro, L. (2007). Câncer de Mama: Prevenção e tratamento. Ediouro.

Mota, S. D. (2011). A atuação do enfermeiro na detecção precoce do Câncer de Mama. Rio de Janeiro.

Salvajoli, J., Souhami, L., \& Faria, S. (2004). Radioterapia em Oncologia. (2. ed.) Rio de Janeiro: MEDSI.

Schwanke, C.H.A.; \& Schneider, R.H. (2008). Atualizações em geriatria e gerontologia: da pesquisa básica à prática clínica. p.163. Porto Alegre: Edipucrs

Silva, M. M.; \& Silva, V. H. (2005) Envelhecimento: importante fator de risco para o câncer. Arquivos Médicos do ABC, Santo André, 30(1), 1118. https://www.portalnepas.org.br/amabc/article/view/273.

Spence, R. A. J.; \& Johnson, P.G. (2012). Oncologia. Guanabara Koogan.

Vieira, C.S., et al.(2012). Oncologia Básica. Fundação Quixote, Teresina, Pi.

\section{Fontes Secundárias}

Alves, P. C.; Sousa, A. P.; Santos, M. C. L.; \& Fernandes, A. F. C. (2010). Conhecimento e expectativas de mulheres no pré-operatório da mastectomia. Revista Escola de Enfermagem - USP. São Paulo.

Ambrósio, D. C. M.; \& Santos, M. A. (2015). Apoio social à mulher mastectomizada: um estudo de revisão. Ciência \& Saúde Coletiva. 20(2), 851-864. Recuperado de https://www.scielosp.org/pdf/csc/2015.v20n3/851-864/pt

Araújo, C. R. G.; Rosas, A. M. M. T. F. (2008). A consulta de enfermagem para clientes e seus cuidadores no setor de radioterapia de hospital universitário. Revista de Enfermagem. Rio de Janeiro.

Balsanelli, A. C. S.; \& GrossI, S. A. A. (2016). Fatores preditores da esperança entre mulheres com câncer de mama durante o tratamento quimioterápico. Rev Esc Enferm USP/SP, 50(6), p.898-904. Recuperado de https:/www.scielo.br/pdf/reeusp/v50n6/pt_0080-6234-reeusp-50-06-00898.pdf

Barreto, R. A. S.; Suzuki, K.; Lima, M. A.; \& Moreira, A. A.(2008). As necessidades de informação de mulheres mastectomizadas subsidiando a assistência de enfermagem. Revista Eletrônica de Enfermagem.

Camargo, C.T .; \& Souza, I .E .O.(2003). Atenção à mulher mastectomizada: Discutindo os aspectos ônticos e a dimensão ontológica da atuação da enfermeira no Hospital do Câncer III. Revista Latino-Americana de Enfermagem. Rio de Janeiro.

Carvalho, J. B.; \& Paes, N. A.(2019). Taxas de mortalidade por câncer corrigidas para os idosos dos estados do Nordeste brasileiro. Ciência \& Saúde Coletiva, 24(10),3857-3866. Recuperado de https://www.scielo.br/pdf/csc/v24n10/1413-8123-csc-24-10-3857.pdf.

Carvalho, C. M. R. G, Brito, C. M. S., Nery I. S., \& Figueiredo, M. L. F. (2009) Prevenção de câncer de mama em mulheres idosas: uma revisão. Revista Brasileira de Enfermagem. Brasília.

Costa, A. I. S.; \& Chaves, M. D.(2012). Dor em pacientes oncológicos sob tratamento quimioterápico. Rev Dor. São Paulo, 13(1) (jan-mar.2012), 45-9. Recuperado de https://www.scielo.br/pdf/rdor/v13n1/a08v13n1.pdf.

Fernandes, P. V.; Iglesiais, A.; \& Avellar, L .Z. (2009). O técnico de enfermagem diante da morte: concepções de morte para técnicos de enfermagem em oncologia e suas implicações na rotina de trabalho e na vida cotidiana. Psicologia Teórica Pratica. 11(11), São Paulo.

Fretta, T. B. et al. (2019). Tratamento de reabilitação para dor em mulheres com câncer de mama. BrJP. São Paulo, 2(3), (jul-set. 2019), 279-83. https://www.scielo.br/pdf/brjp/v2n3/pt_2595-0118-brjp-02-03-0279.pdf.

Leal, J. H. S.; Cubero, D.; \& Giglio, A .D. (2010). Hormonio terapia paliativa em câncer de mama: aspectos práticos e revisão da literatura. Revista Brasileira de Clínica Medica. São Paulo. p.338-343.

Maia, L. F. S. (2008). Sistematização da Assistência de Enfermagem Perioperatória: uma revisão de literatura. Revista Escola de Enfermagem São Paulo.

Melo, E .M.; Araújo, T.L.; Oliveira, T.C.; \& Almeida, D.T. (2002). Mulher mastectomizada em tratamento quimioterápico: um estudo dos comportamentos na perspectiva do modelo adaptativo de ROY. Revista Brasileira de Cancerologia. Brasília.

Mineo, F. L. V. et al. (2013). Assistência de enfermagem no tratamento do câncer de mama. Revista Eletrônica Gestão \& Saúde ISSN:1982-4785. periodicos.unb.br/index.php/rgs/article/download/256/244. 
Recco, D. C.; Luiz, B. C.; \& Pinto, M. H. (2005). O cuidado prestado ao paciente portador de doença oncológica: na visão de um grupo de enfermeiras de um hospital de grande porte do interior do estado de São Paulo. Arquivo Ciência Saúde. São Paulo.

Sá, L. R.; \& Souza, I. E. O. (2010). Enfermagem em saúde da mulher: Re-visitando a produção acadêmica sobre o câncer de mama. Revista Pesquisa Cuidado Fundamental [online]. Rio de Janeiro.

Santos, M. C. L.; Sousa, F. S.; Alves, P. C.; Bonfim, I. M.; \& Fernandes, A. F. C. (2011) Comunicação terapêutica no cuidado pré-operatório de mastectomia. Revista Brasileira de Enfermagem. 63(4), Brasília.

Sartori, A. C. N.; \& Basso, C .S. (2019). Câncer de mama: uma breve revisão de literatura. Perspectiva, Erechim. 43(161) (mar/2019), 07-13. https://www.uricer.edu.br/site/pdfs/perspectiva/161_742.pdf.

Souza, V. O.; Grando, J. P. S.; \& Couto Filho, J. O. (2008). Tempo decorrido entre o diagnóstico de câncer de mama e o início do tratamento, em pacientes atendidas no Instituto de Câncer de Londrina (ICL). RBM Rev Bras Med, 65(5), (jun.2008). Recuperado de http://bases.bireme.br/cgibin/wxislind.exe/iah/online/?IsisScript=iah/iah.xis\&src=google\&base=LILACS\&lang=p\&nextAction=lnk\&exprSearch=485556\&indexSearch=ID

Stumm, E. M .F.; Leite, M. T.; \& Maschio G. (2008). Vivências de uma equipe de enfermagem no cuidado a pacientes com câncer. Cogitare Enfermagem. Rio Grande do Sul.

TNM (2004). Classificação de Tumores Malignos. Recuperado de https://bvsms.saude.gov.br/bvs/publicacoes/inca/tnm2.pdf

Vieira, C. P.; Lopes, M. H. M.; \& Shimo. A. K .K.(2007). Sentimentos e experiências na vida das mulheres com câncer de mama. Revista Escola de Enfermagem - USP, São Paulo.

\section{Documental}

Brasil (1987). Conselho Federal de Enfermagem - COFEN. Decreto no 94.406/87 de 8 junho de 1987. http://www.cofen.gov.br/decreto-n-9440687_4173.html.

Brasil (1998). Conselho Federal de Enfermagem - COFEN. Resolução COFEN-210/1998 de 1 de julho de 1998. Aprova as Normas Técnicas de Biossegurança Individual, Coletiva e Ambiental dos procedimentos a serem realizadas pelos profissionais de Enfermagem que trabalham com quimioterapia antineoplásica. Recuperado de http://www.cofen.gov.br/resoluo-cofen-2101998_4257.html.

Brasil (1998). Conselho Federal de Enfermagem- COFEN. Resolução COFEN-211/1998 de 1 de julho de 1998. Aprova as Normas Técnicas de radioproteção nos procedimentos a serem realizados pelos profissionais de Enfermagem que trabalham com radiação ionizante em Radioterapia, Medicina Nuclear e Serviços de Imagem. http://www.cofen.gov.br/resoluo-cofen-2111998_4258.html

Brasil (2002). Instituto Nacional de Câncer - INCA. Comitê de Padronizações. A radioterapia e você.

Brasil (2020). Instituto Nacional de Câncer José Alencar Gomes da Silva. Estimativa 2020: Incidência do câncer no Brasil. Rio de Janeiro: INCA. https://www.inca.gov.br/sites/ufu.sti.inca.local/files//media/document//..

Brasil (2003). Conselho Federal de Enfermagem - COFEN. Código de Deontologia dos Profissionais de Enfermagem. Rio de Janeiro: Gráfica do COREN/RJ.

Brasil (2008). Ministério da Saúde. Ações de enfermagem para o controle do câncer: Uma proposta de integração ensino-serviço. (3a ed.) p.256-271.

Brasil (2009). Conselho Federal de Enfermagem- COFEN. Resolução COFEN-358/2009 de 15 de outubro de 2009. Dispõe sobre a Sistematização da Assistência de Enfermagem e a implementação do Processo de Enfermagem em ambientes, públicos ou privados, em que ocorre o cuidado profissional de Enfermagem, e dá outras providências. Recuperado de http://www.cofen.gov.br/resoluo-cofen-3582009_4384.html.

Brasil (2010). Ministério da Saúde. Instituto Nacional do Câncer (INCA). Estimativas de incidência e mortalidade por câncer no Brasil. http://bvsms.saude.gov.br/bvs/publicacoes/estimativa_2010_incidencia_cancer.pdf.

Brasil (2011). Instituto Nacional de Câncer. Encontro Internacional sobre Rastreamento do Câncer de Mama: resumo das apresentações. Caderno resumo, Rio de Janeiro, INCA. Recuperado de http://www.inca.gov.br.

Brasil (2013). Ministério da Saúde, Portaria $n^{o} .874$ de 16 de maio de 2013. Institui a Política Nacional para a Prevenção e Controle do Câncer na Rede de Atenção à Saúde das Pessoas com Doenças Crônicas no âmbito do Sistema Único de Saúde (SUS). Recuperado de https://bvsms.saude.gov.br/bvs/saudelegis/gm/2013/prt0874_16_05_2013.html.

Brasil (2015). Ministério da Saúde. Diretrizes para a detecção precoce do câncer de mama no Brasil / Instituto Nacional de Câncer José Alencar Gomes da Silva - Rio de Janeiro: INCA, 168 p.: il. color. https://www.inca.gov.br/sites/ufu.sti.inca.local/files//media/document//diretrizes_deteccao_ precoce_cancer_mama_brasil.pdf.

Gil, A. C.(2002). Como elaborar projetos de pesquisa. São Paulo, SP: Atlas.

International Agency for Research on Cancer(2020). Cancer today. Lyon: Who. Recuperado de https://gco.iarc.fr/today/home.

Marconi, M. A. \& Lakatos, E. M. (2010). Fundamentos de metodologia científica. 7. ed. São Paulo: Atlas. 